\title{
Perspectives on achieving institutional trust in personalized medicine
}

\section{Samuel, Dheensa}

UAJB-2017-0693

Kraft and colleagues have provided an interesting discussion on the inadequacy of consent and importance of institutional trustworthiness for personalized medicine initiatives, particularly with relation to ethnically diverse patients. These authors suggest three ways to work towards achieving institutional trustworthiness for such patients: addressing the role of history and experience on trust; engaging concerns about potential group harm; and addressing cultural values and communication barriers, and integrating patient values and expectations into oversight and governance structures.

We use this response to bring to the fore wider scholarship and activities that have already explored issues of institutional trust in detail. We set the scene by highlighting previous scholarship that has problematized consent and called for a more trusting approach to research/clinical practice; including a UK personalized medicine initiative which is currently working towards building such trust. Then, given, as Kraft and colleagues state, that problems still remain regarding how to build such trust in practice, particularly with relation to ethnically diverse patients, we discuss how a solidarity-based model offers one approach to help, especially with respect to Kraft and colleagues' point about the need to engage with concerns about potential harm inflicted on these patients.

First, Kraft and colleagues' work adds to an already increasing body of social science and anthropology literature which questions the adequacy of informed consent as a proxy for ethical medical/research practice (O’Neill, 2002), including that of personalized medicine (Samuel et al, 
2017). This literature particularly problematizes the role of consent as a tool for information provision (Koening, 2014). It notes that decision-making during consent is not always a rational weighing up of harms and benefits about the research/care being undertaken; and providing more and more information about risks and benefits does not make consent more 'ethical'. Rather, consent is relational - alongside the need for information, it is also about personal, emotional, cultural, social, and economic circumstances, and, importantly for this paper, about the institutional context (and its perceived trustworthiness) within which consent is received (Samuel et al, 2017). As many of these scholars have long noted, and as Kraft and colleagues point out for personalized medicine practice, to create an ethical programme we therefore need to move away from solely relying on notions of consent, but also consider other ethical principles alongside.

These issues have been discussed in the UK. Here, the UK Chief Medical Officer's 2016 Annual Report, Generation Genome focused on a vision to fully integrate personalized medicine into all aspects of the UK's National Health Service (NHS) and this shift is currently being spring-boarded via the 100,000 Genomes Project (100kGP). 100kGP is sequencing 100,000 genomes from consenting NHS patients/family members who have a rare disease, an infectious disease, or cancer, to analyse their genomes for clinical diagnosis; offer further (unconnected) health-related results on request; as well as placing the genomes in a research bank with other patient health data, as a future resource for research projects. The legacy aim of 100kGP is integrated personalized medicine in the NHS which routinely offers research participation/biobanking to patients who have relevant diagnostic investigations, which they may refuse with no detriment to treatment.

In line with Kraft and colleagues' work, we have conducted interviews with participants/patients who have taken part in $100 \mathrm{kGP}$. Our research shows that interviewees take part not only because 
they are reassured by the consent process, but because they envisage the NHS as the 'face' of the project: patients viewed the NHS as trustworthy, and interviewees placed a large amount of trust in the NHS to handle their genomes responsibly and for clinicians to have their best interests at heart (Dheensa et al., 2016; Dheensa and Fenwick, forthcoming).

These findings reflect a large amount of thought by Genomics England (deliverer of 100kGP), associated individuals and wider organisations, which has gone into ensuring that the implementation of 100kGP, and personalized medicine in the UK more broadly, occurs in an ethically and socially acceptable manner which goes beyond (but still includes) notions of consent and, importantly, includes notions of deserving trust. Indeed, for Genomics England, being institutionally trustworthy is equally important to providing best practice consent processes, and our research has shown that part of this has been achieved by presenting the public image of the organisation in an ethically responsible manner (Samuel and Farsides, 2017a), and also by ensuring that public expectations are appropriately managed (Samuel and Farsides, 2017b).

Issues relating to trust do remain, and there is still much work being conducted at Genomics England and elsewhere to understand patient/participant perspectives on taking part in 100kGP, and how this relates to notions of trust, but much effort has been invested into working through these issues. One example is the establishment of a Participant Panel which advise Genomics England; another is having an Ethics working group exploring issues related to ethnically diverse patients, comprised of 50/50 patients and professionals.

This emphasis on trust is also reflected in the final Chapter of the Generation Genome, which stresses the need to create an appropriate future ethical governance system (an 'ethical ecosystem') to support the wider uptake of genomic medicine in England, that can be trusted by 
patients; responsible health professionals which uphold this trust; clear, open protection, and privacy of genomic data; and best practice approaches to patient consent especially in the context of managing uncertainty (Lucassen et al. 2017).

Given the ongoing work to build patient/participant trust in existing personalized medicine initiatives, it is important to explore the perspectives of others who have considered the issue. In this final section we draw on the ethical approach of solidarity as a way to conceptualize institutional trust, and we show how this approach can offer one way to address Kraft and colleagues' point about the need to engage with concerns about potential harm inflicted on ethnically diverse patients, as discussed in their article.

In the solidarity-based approach, which has been discussed mostly in relation to biobanking, but also for personalized healthcare, an individual under 'board consent' provides health data to an institution knowing that there are risks and uncertainty attached to what future research will be conducted on their data (Prainsack and Buyx, 2013; Prainsack, 2017). This is because the solidarity approach places strong ethical emphasis on people's willingness to engage in activities which may carry some risks (i.e., donating their genomic/heath data for uncertain uses with small risks attached) for the benefit of others. This fits with some of the findings from the 100kGP in terms of rare disease patients and family members - that they wanted to take part to benefit someone else.

Proponents explain that in this model, individual autonomy, and the requirement for specific information to be given to a person about how their health data will be used, along with any associated risks, prior to consent, is not considered the dominant ethical model. Rather, consent is based on a person understanding, and signing up to, the broad value-base mission statement of 
the specific research institution collecting their health data i.e., what type of research the institution will conduct; what ethical systems are in place to ensure this; how the institution addresses openness, transparency, public education/engagement. The participant must then place a certain amount of trust in the institution to uphold this value base, and the institution must live up to it (and put good governance models in place to ensure this). This model therefore places the responsibility on institutions to be transparent about their own stakes and interests (including commercial ones, if applicable), and about the types of harms that may not be foreseeable.

One way to uphold this trust is, say solidarity scholars, by making sure that if harm occurs, affected people are adequately supported. Arguing that legal remedies currently available are not sufficient, they argue for making funds available to provide financial support to individuals who can make plausible that they have been harmed as a result of donating their data - without them having to prove wrongdoing on the side of the biobank (Prainsack, 2017). In this model, therefore, there is a shift from emphasising only risk management towards also improving harm mitigation. In other words, rather than pretending to foresee all the different risks attached to personalised medicine (for example, discrimination), which is increasingly difficult to do in the era of datadriven research, proponents argue, we should "increase the scope for action after an undesirable event has taken place" (Prainsack and Buyx, 2013: 83).

Adopting this approach could be one way to instil trust in a personalized medicine initiative, particularly for minority group patients. This is because if an open and public system is established, and actually functioned to allow those discriminated against to be financially supported, and people were made aware of it and could see it in action, this might improve the institution's perceived trustworthiness. 
In conclusion, we have highlighted how- in line with Kraft and colleagues findings and other research - personalized medicine in the UK, spring-boarded by $100 \mathrm{kGP}$, is placing the need for institutions to be trustworthy as a high priority. We have highlighted the solidarity-based perspective as one way this can be achieved, particularly for minority group patients.

\section{REFERENCES}

1. Dheensa, S., A. Fenwick, A. Lucassen, and G. Crawford. "The UK 100,000 genomes project: views, expectations, and experiences of the first patients recruited". Presented at European Society of Human Genetics, Barcelona, May 21-24 2016, http://bit.ly/2BB6sJt

2. Koenig, B. A. "Have we asked too much of consent?." Hastings Center Report 44, no. 4 (2014): 33-34.

3. Kraft and colleagues FULL REFERENCE HERE "Beyond Consent: Building Trusting Relationships with Diverse Populations in Precision Medicine Research" (2018). AJOB

4. Lucassen, A., J. Montgomery, and M. Parker. "Ethics and the Social Contract". In Generation Genome, edited by S. Davies. Annual report of the Chief Medical Officer 2016: Generation Genome." Chap16 p1-16. London: Department of Health. 2017. https://www.ncbi.nlm.nih.gov/books/NBK355264/

5. O’Neill, O. "Autonomy and trust in Bioethics" (2002). Cambridge University Press. UK

6. Prainsack, B., and Buyx, A. "The solidarity-based approach to the governance of research biobanks" Medical Law Review (2103). 21:71-91

7. Prainsack B. 2017. Research for personalised medicine: Time for solidarity. Medicine and Law 36/1: 87-98.

8. Samuel, G. N, and B. Farsides. "Public trust and 'ethics review' as a commodity: the case of Genomics England Limited and the UK's 100,000 genomes project." Medicine, Health Care 
and Philosophy (2017a): 1-10. https://link.springer.com/content/pdf/10.1007\%2Fs11019017-9810-1.pdf

9. Samuel, G. N, and B. Farsides. "Genomics England's implementation of its public engagement strategy: Blurred boundaries between engagement for the United Kingdom's 100,000 Genomes project and the need for public support" (2017b): http://journals.sagepub.com/doi/pdf/10.1177/0963662517747200

10. Samuel, G. N., S. Dheensa, B. Farsides, A. Fenwick, and A. Lucassen. "Healthcare professionals' and patients' perspectives on consent to clinical genetic testing: moving towards a more relational approach." BMC Medical Ethics 18, no. 1 (2017): 47. 